Y. Yamada

Nagoya Math. J.

Vol. 73 (1979), 69-98

\title{
ON THE DECAY OF SOLUTIONS FOR SOME NONLINEAR EVOLUTION EQUATIONS OF SECOND ORDER
}

\author{
YOSHIO YAMADA
}

\section{Introduction}

In this paper we consider nonlinear evolution equations of the form

$$
u^{\prime \prime}(t)+A u(t)+B(t) u^{\prime}(t)=f(t), \quad 0 \leqq t<\infty,
$$

$\left(u^{\prime \prime}(t)=d^{2} u(t) / d t^{2}, u^{\prime}(t)=d u(t) / d t\right)$, where $A$ and $B(t)$ are (possibly) nonlinear operators. Various examples of equations of type (E) arise in physics; for instance, if $A u=-\Delta u$ and $B(t) u^{\prime}=\left|u^{\prime}\right| u^{\prime}$, the equation represents a classical vibrating membrane with the resistance proportional to the velocity. As to the initial value problems for $(\mathrm{E})$, many authors have established various sufficient conditions on $A$ and $B(t)$ so that there exists a global solution of (E) (see e.g. Barbu [4], Lions [10] and Tsutsumi [18]).

As for the asymptotic behavior of solutions of (E), Nakao [14], [15] has recently obtained interesting results under the appropriate assumptions on $A$ and $B(t)$; in [15] he treats the case when $A$ as well as $B(t)$ depends on $t$. When $f(t)$ tends to zero (in some sense) as $t \rightarrow \infty$, he showed that any global solution of (E) satisfying the energy equation satisfies a difference inequality of the form

$$
\sup _{t \leqq s \leqq t+1} E(s)^{1+\alpha} \leqq C(t+1)^{r}\{E(t)-E(t+1)\}+g(t),
$$

where $E(t)$ denotes the energy associated with such a solution, $g(t)$ is a nonnegative function tending to zero as $t \rightarrow \infty$ and $\alpha, \gamma$ and $C$ are some positive constants. From this inequality he derived the decay property of $E(t)$ : if $f(t)$ tends to zero in an appropriate sense as $t \rightarrow \infty$, then $E(t)$ decays to zero as $t \rightarrow \infty$.

The main purpose of the present paper is to discuss the asymptotic behavior of solutions of (E). We put almost the same assumptions on

Received October 15, 1977. 
$A$ and $B(t)$ as those of Nakao [15] so that the decay property of (E) holds; but, for simplicity of arguments, the case when $A$ depends on $t$ is not treated here. Moreover, we assume that there exists a global solution of (E) which satisfies a certain kind of an energy inequality (see (2.6)). Then our problem is the following: if $f(t)$ tends to zero as $t \rightarrow \infty$, how fast does the energy $E(t)$ decay to zero as $t \rightarrow \infty$ ? Our approach to this problem relies on the weighted energy method and is quite different from Nakao's. In order to investigate the rate of the decay of $E(t)$, we introduce a pair of $\{G(t), h(t)\}$ of two monotone increasing functions on $[0, \infty)$ which satisfy several conditions (see $\S 2.4$ ). We take $G(t)$ as a weight function of $E(t)$ and take $h(t)$ as an auxiliary function of $G(t)$. Using this pair $\{G(t), h(t)\}$, we can show

$$
\sup _{t \geqq 0} G(t) E(t)<\infty
$$

If the rate of the convergence of $f(t)$ to zero is known, we can obtain, with a suitable choice of $\{G(t), h(t)\}$, preciser results on the rate of the decay of $E(t)$ than those of Nakao [14], [15].

The content of this paper is as follows. We introduce some notation in $\S 2.1$ and give some assumptions on $A$ and $B(t)$ in $\S 2.2$. In $\S 2.3$ we define a solution of $(\mathrm{E})$ which has the weak conservation property. Main results (Theorems 1, 2 and 3) are stated in $\S 2.4$. $\S 3$ is devoted to the proofs of Theorems 1,2 and 3. Finally, in $\S 4$ we apply our results to some nonlinear partial differential equations such as the nonlinear wave equations and the nonlinear beam equations.

\section{§2. Assumptions and Results}

\subsection{Preliminaries}

We first of all explain some notation which will be used later.

Let $X$ be a real Banach space. We denote the norm in $X$ by $|\cdot|_{X}$ and denote the dual space of $X$ by $X^{*}$. The pairing between $x^{*} \in X^{*}$ and $x \in X$ is denoted by $\left(x^{*}, x\right)_{X}$.

Let $1 \leqq r<\infty$ and $a \leqq b$. When $X$ is a real Banach space, we say that $f$ belongs to $L^{r}(a, b ; X)$ if $f$ is measurable in $a \leqq t \leqq b$ with values in $X$ and satisfies

$$
|f|_{L^{r}(a, b ; X)}<\infty
$$


where

$$
\begin{aligned}
|f|_{L r(a, b ; X)} & =\left(\int_{a}^{b}|f(t)|_{X}^{r} d t\right)^{1 / r} & & \text { if } 1 \leqq r<\infty, \\
& =\operatorname{ess~sup~}_{a \leqq t \leqq b}|f(t)|_{X} & & \text { if } r=\infty .
\end{aligned}
$$

In particular, for $X=\boldsymbol{R}$ we simply write $L^{r}(a, b)=L^{r}(a, b ; R)$. Finally, we define $L_{\mathrm{loc}}^{r}(0, \infty ; X)$ by

$$
L_{1 \mathrm{loc}}^{r}(0, \infty ; X)=\left\{f:[0, \infty) \rightarrow X ; f \in L^{r}(0, T ; X) \text { for any } T>0\right\} .
$$

\subsection{Assumptions}

We are given a real Hilbert space $H$ and two real Banach spaces $V$ and $W$ which satisfy

$$
V \subset W \subset H
$$

where each inclusion mapping is continuous. Then there exist positive constants $c_{1}$ and $c_{2}$ such that

$$
|v|_{W} \leqq c_{1}|v|_{V} \quad \text { for } v \in V
$$

and

$$
|w|_{H} \leqq c_{2}|w|_{W} \quad \text { for } w \in W
$$

Moreover, we assume that $V$ is dense in $W$ and $H$. We identify $H$ with its own dual, so that the following inclusion relation holds

$$
V \subset W \subset H \subset W^{*} \subset V^{*} .
$$

The natural pairing between $u^{*} \in V^{*}$ (resp. $u^{*} \in W^{*}$ ) and $u \in V$ (resp. $u \in W)$ is denoted by $\left(u^{*}, u\right)_{V}$ (resp. $\left.\left(u^{*}, u\right)_{W}\right)$; if $u^{*}, u \in H$, this is the ordinary inner product $\left(u^{*}, u\right)_{H}$ in $H$.

Besides these spaces we are given two nonlinear operators $A$ and $B(t)$ which satisfy the following assumptions.

(A.1) $A: V \rightarrow V^{*}$ is the Fréchet derivative of a convex functional $F_{A}$ on $V$ such that

$$
F_{A}(0)=0
$$

and

$$
F_{A}(u) \geqq a|u|_{V}^{p} \quad \text { for } u \in V \text {, }
$$


where $a>0$ and $p \geqq 2$.

(A.2) For each $0 \leqq t<\infty, B(t): W \rightarrow W^{*}$ satisfies

$$
(B(t) u, u)_{W} \geqq b_{1} \beta(t)|u|_{W}^{q} \quad \text { for } u \in W,
$$

and

$$
\left|(B(t) u, v)_{W}\right| \leqq b_{2} \beta(t)|u|_{W}^{q-1}|v|_{W} \quad \text { for } u, v \in W,
$$

where $b_{2} \geqq b_{1}>0, q \geqq 2$ and $\beta(t)$ is a positive continuous function on $[0, \infty)$.

Finally we assume

$$
f \in L^{\infty}\left(0, \infty ; W^{*}\right) \text {. }
$$

Remark 2.1. Assumption (A.1) implies

$$
(A u, u)_{V} \geqq F_{A}(u) \geqq a|u|_{V}^{p} \quad \text { for } u \in V \text {. }
$$

Indeed, since, by the convexity of $F_{A}$,

$$
\alpha\left(F_{A}(0)-F_{A}(u)\right) \geqq F_{A}(u+\alpha(0-u))-F_{A}(u), \quad 0<\alpha<1, u \in V,
$$

we conclude, by dividing both sides of the above inequality by $\alpha$ and letting $\alpha \downarrow 0$, that (2.3) holds.

Remark 2.2. We can replace (A.1) with a slightly weaker assumption $(\text { A.1 })^{\prime}$ :

(A.1) $\quad A: V \rightarrow V^{*}$ is the Fréchet derivative of a convex functional $F_{A}$ on $V$ such that

$$
F_{A}(0)=0
$$

and

$$
F_{A}(u) \geqq a_{1}|u|_{V_{1}}^{p} \quad \text { for } u \in V \text {, }
$$

where $a_{1}>0, p \geqq 2$ and $V_{1}$ is a real Banach space such that $V \subset V_{1} \subset W$ and the inclusion mapping of $V_{1}$ into $W$ is continuous.

In what follows, we treat only the case of (A.1) because the arguments in the case of (A.1)' are quite similar (see Remark 3.1).

\subsection{Definitions}

Under assumptions (A.1)-(A.3) we consider abstract nonlinear evolu- 
tion equations of the form

$$
u^{\prime \prime}(t)+A u(t)+B(t) u^{\prime}(t)=f(t), \quad 0 \leqq t<\infty,
$$

where $u^{\prime \prime}(t)=d^{2} u(t) / d t^{2}$ and $u^{\prime}(t)=d u(t) / d t$. We first define a class of solutions of $(E)$ in which we investigate $(E)$.

DeFinition 2.1. Let $u:[0, \infty) \rightarrow V$. Then $u$ is called a solution of (E) if $u \in L_{\mathrm{loc}}^{\infty}(0, \infty ; V), u^{\prime} \in L_{\mathrm{loc}}^{\infty}(0, \infty ; H) \cap L_{\mathrm{loc}}^{q}(0, \infty ; W)$ and, for every such function $\phi:[0, \infty) \rightarrow V$, $u$ satisfies

$$
\begin{aligned}
&\left(u^{\prime}(t), \phi(t)\right)_{H}+\int_{0}^{t}\{-\left(u^{\prime}(s), \phi^{\prime}(s)\right)_{H}+(A u(s), \phi(s))_{V} \\
&\left.+\left(B(s) u^{\prime}(s), \phi(s)\right)_{W}\right\} d s \\
&=\left(u^{\prime}(0), \phi(0)\right)_{H}+\int_{0}^{t}(f(s), \phi(s))_{V} d s, \quad 0 \leqq t<\infty .
\end{aligned}
$$

Definition 2.2. Let $u$ be a solution of (E). Then the energy $E(t)$ is defined by

$$
E(t)=\frac{1}{2}\left|u^{\prime}(t)\right|_{H}^{2}+F_{A}(u(t)) .
$$

Moreover, it is called that $u$ has the weak conservation property if, for any positive $C^{1}$-function $\psi$ on $[0, \infty), u$ satisfies

$$
\begin{aligned}
& \psi(t) E(t)+\int_{s}^{t} \psi(r)\left(B(r) u^{\prime}(r), u^{\prime}(r)\right)_{W} d r \\
& \leqq \psi(s) E(s)+\int_{s}^{t} \psi^{\prime}(r) E(r) d r+\int_{s}^{t} \psi(r)\left(f(r), u^{\prime}(r)\right)_{W} d r, \\
& 0 \leqq s \leqq t .
\end{aligned}
$$

In this paper we are interested in the dacay property of solutions of (E) rather than the existence of solutions of (E). Therefore, in what follows, we always assume the existence of a solution of (E) with the weak conservation property and treat only such a solution.

For the existence of solutions of (E) with the weak conservation property, we refer to Barbu [4], Brezis [5], Lions and Strauss [11], Strauss [17], Tsutsumi [18] and Yamada [19] (see also §4).

Remark 2.3. Formally, setting $\phi=u^{\prime}$ in (2.4) leads to the energy equation of the form 


$$
\begin{array}{r}
E(t)+\int_{0}^{t}\left(B(s) u^{\prime}(s), u^{\prime}(s)\right)_{W} d s=E(0)+\int_{0}^{t}\left(f(s), u^{\prime}(s)\right)_{W} d s, \\
0 \leqq t<\infty .
\end{array}
$$

If (2.7) holds, then (2.6) also holds for any positive $C^{1}$-function $\psi$ on $[0, \infty)$. Therefore, any solution of (E) satisfying the energy equation (2.7) has the weak conservation property.

\subsection{Results}

Now we shall investigate the rate of the decay to zero of solutions of (E) when $f(t)$ tends to zero as $t \rightarrow \infty$. To this end, we employ the weighted energy method. We assume that we can take a pair of two functions $G(t)$ and $h(t)$ which satisfy the following conditions.

(GH.1) $G \in C[0, \infty) \cap C^{2}(0, \infty)$ is strictly monotone increasing on $[0, \infty)$ and satisfies

$$
G(0) \geqq 0 \text { and } \lim _{t \rightarrow \infty} G(t)=\infty .
$$

(GH.2) $h \in C[0, \infty)$ is strictly monotone increasing on $[0, \infty)$ and satisfies

$$
t>h(t)>0 \quad \text { for } t>t_{0} \text { and } \lim _{t \rightarrow \infty} h(t)=\infty,
$$

where $t_{0}$ is a non-negative constant.

$$
\sup _{t>t_{0}}\left\{\left|\beta^{-1 / q} \cdot G^{1 / q^{\prime}} \cdot f\right|_{L^{q^{\prime}\left(h(t), t ; W^{*}\right)}}\right\} \equiv L<\infty,
$$

where $q^{\prime}=q /(q-1)$ and $\beta$ is the function in (A.2).

In addition to (GH.1)-(GH.3), we put different conditions on $\{G, h\}$ according as $p+q>4$ or $p=q=2$. (Recall that $p(\geqq 2)$ and $q(\geqq 2)$ are constants in (A.1) and (A.2), respectively.)

When $p+q>4$, we set the following conditions on $\{G, h\}$.

(GH.4) There exists a positive constant $M$ with the following properties (i), (ii) and (iii).

$$
\text { (i) } \begin{aligned}
k_{1}(p)+k_{2}(p) \equiv & \frac{c_{1} c_{2}}{2^{1 / 2} a^{1 / p}}\left[\operatorname { s u p } _ { t > t _ { 0 } } \left\{\left|G^{\prime \prime} \cdot\left(G^{\prime}\right)^{-(p+2) /(2 p)}\right|_{\left.L^{2 p /(p-2)(h(t), t)}\right\}}\right.\right. \\
& \left.+2\left|G^{\prime} \cdot G^{-(p+2) /(2 p)}\right|_{L^{\infty}\left(h\left(t_{0}\right), \infty\right)}\right] \\
< & M^{(p-2) /(2 p)} .
\end{aligned}
$$


(ii) $\quad k_{3}(q) \equiv \frac{3 c_{2}^{2}}{2 b_{1}^{2 / q}} \sup _{t>t_{0}}\left\{\left|G^{\prime} \cdot(\beta G)^{-2 / q}\right|_{L q /(q-2)(h(t), t)}\right\}<M^{(q-2) / q}$.

(iii) If $p \geqq q$, then

$$
\begin{aligned}
k_{4}(p, q) & \equiv \frac{b_{2} c_{1}}{a^{1 / p} b_{1}^{(q-1) / q}} \sup _{t>t_{0}}\left\{\left|\beta^{1 / q} \cdot\left(G^{\prime}\right)^{(p-1) / p} \cdot G^{-(q-1) / q}\right|_{\left.L^{p q /(p-q)(h(t), t)}\right\}}\right\} \\
& <M^{(p-q) /(p q)} .
\end{aligned}
$$

If $p<q$, then

$$
\lim _{t \rightarrow \infty}\left\{\beta(t) G^{\prime}(t)^{q-1} G(t)^{-(q-1)}\right\}=0 .
$$

Here $a, b_{1}, b_{2}, c_{1}$ and $c_{2}$ are positive constants in $\S 2.2$.

(GH.5) $\quad\left|G(h(\cdot)) G(\cdot)^{-1}\right|_{L^{\infty}\left(t_{0}, \infty\right)}< \begin{cases}1 & \text { if } p>2 \\ 1-\frac{k_{2}(2)}{1-k_{1}(2)} & \text { if } p=2 .\end{cases}$

When $p=q=2$, we replace conditions (GH.4) and (GH.5) with the following conditions (GH.4) ${ }^{\prime}$ and (GH.5)'.

$(\mathrm{GH} .4)^{\prime}$

$$
k_{1}(2)+k_{2}(2)+k_{3}(2)+k_{4}(2,2)<1 .
$$

$(\mathrm{GH} .5)^{\prime} \quad\left|G(h(\cdot)) G(\cdot)^{-1}\right|_{L^{\infty}\left(t_{0}, \infty\right)}<1-\frac{k_{2}(2)}{1-k_{1}(2)-k_{3}(2)-k_{4}(2,2)}$.

When $\beta \equiv 1$ and $f \equiv 0$, examples of $\{G, h\}$ which satisfy the above conditions are given by functions of the form

$$
\begin{array}{ll}
\{G(t), h(t)\}=\left\{t^{\alpha}, c t\right\}, \quad \alpha>0,1>c>0, & \text { if } p+q>4, \\
\{G(t), h(t)\}=\left\{e^{\alpha t}, t-c\right\}, \quad \alpha>0, c>0, & \text { if } p=q=2,
\end{array}
$$

where $\alpha$ and $c$ in (2.8) (resp. (2.9)) must be determined so as to satisfy (GH.4) and (GH.5) (resp. (GH.4) and (GH.5)'). see also $\S \S 3.3$ and 3.4 .

Now we shall state our main theorems.

THEOREM 1. Let $u$ be a solution of (E) with the weak conservation property. If $\{G, h\}$ satisfies (GH.1)-(GH.5) in case $p+q>4$ or (GH.1)(GH.3), (GH.4)' and (GH.5)' in case $p=q=2$, then there exists a positive constant $C$ such that

$$
G(t) E(t) \leqq C, \quad t \geqq 0,
$$

where $E(t)$ is the energy defined by (2.5). 
Remark 2.4. Let $p<q$. If it is known that $E(t)$ decays to zero as $t \rightarrow \infty$, then the latter condition in (GH.4) (iii) can be replaced with the weaker one

$$
\left|\beta \cdot\left(G^{\prime}\right)^{q-1} \cdot G^{-(q-1)}\right|_{L^{\infty}\left(h\left(t_{0}\right), \infty\right)}<\infty
$$

(cf. Remark 3.2).

When $\beta(t)=(t+1)^{\theta}(-1 \leqq \theta \leqq q-1)$ and $|f(t)|_{W^{*}} \rightarrow 0$ fast enough, we have the following result on the rate of the decay of $E(t)$.

THEOREM 2. Let $u$ be a solution of (E) with the weak conservation property. If $\beta(t)=(t+1)^{\theta}$ with $-1 \leqq \theta \leqq q-1$ and $|f(t)|_{W^{*}}=0\left(t^{-r}\right)$ with $\gamma>(q-1-\theta) / q$ as $t \rightarrow \infty$, then the following results hold.

(i ) When $-1<\theta<q-1$,

$$
E(t)=0\left(t^{-\alpha}\right) \quad(\text { as } t \rightarrow \infty), \alpha=\min \left\{\delta, \frac{q \gamma-q+1+\theta}{q-1}\right\},
$$

where $\delta$ is a positive number defined by

$$
\delta= \begin{cases}\frac{p(q-1-\theta)}{p-q} & \text { if }(p-2)(1+\theta)>p(q-2) \\ \frac{2(1+\theta)}{q-2} & \text { if }(p-2)(1+\theta) \leqq p(q-2) .\end{cases}
$$

(ii) When $\theta=q-1$,

$$
E(t)=\left\{\begin{array}{lll}
0\left((\log t)^{-p(q-1) /(p-q)}\right) & (\text { as } t \rightarrow \infty) & \text { if } p>q \\
0\left(t^{-\alpha}\right) & (\text { as } t \rightarrow \infty) & \text { if } p \leqq q,
\end{array}\right.
$$

where $\alpha$ is a positive number such that

$$
\begin{cases}\alpha \leqq \min \left\{\frac{2 q}{q-2}, \frac{q \gamma}{q-1}\right\} \quad \text { and } \quad \alpha<\frac{a^{1 /(p-1)} b_{1}}{\left(b_{2} c_{1}\right)^{p /(p-1)}} & \text { if } p=q \\ \alpha=\min \left\{\frac{2 q}{q-2}, \frac{q \gamma}{q-1}\right\} & \text { if } p<q .\end{cases}
$$

(iii) When $\theta=-1$,

$$
E(t)=\left\{\begin{array}{lll}
0\left((\log t)^{-2 /(q-2)}\right) & (\text { as } t \rightarrow \infty) & \text { if } q>2 \\
0\left(t^{-\alpha}\right) & (\text { as } t \rightarrow \infty) & \text { if } q=2,
\end{array}\right.
$$

where $\alpha$ is a positive number satisfying 


$$
\alpha \leqq \min \left\{\frac{2 p}{p-2}, 2 \gamma-2\right\} \quad \text { and } \quad \alpha<\frac{2 b_{1}}{3 c_{2}^{2}}
$$

In particular, when $p=q=2$ and $|f(t)|_{W^{*}}$ decays to zero exponentially, we have

THEOREM 3. Let $u$ be a solution of (E) with the weak conservation property. If $p=q=2, \beta(t)=(t+1)^{\theta}$ with $-1<\theta<1$ and $|f(t)|_{W^{*}}$ $=0\left(\exp \left(-\gamma t^{1-|\theta|}\right)\right)$ with $\gamma>0$ as $t \rightarrow \infty$, then

$$
E(t)=0\left(\exp \left(-\alpha t^{1-|\theta|}\right)\right) \quad \text { as } t \rightarrow \infty,
$$

where $\alpha$ is a positive number satisfying

$$
\begin{cases}\alpha \leqq 2 \gamma \quad \text { and } \quad \alpha<\frac{a b_{1}}{b_{2}^{2} c_{1}^{2}(1-\theta)} & \text { if } 0<\theta<1 \\ \alpha \leqq 2 \gamma \quad \text { and } 3\left(\frac{c_{1} c_{2}}{\sqrt{2 \alpha}}+\frac{c_{2}^{2}}{2 b_{1}}\right) \alpha+\frac{b_{2} c_{1}}{\sqrt{a b_{1}}} \sqrt{\alpha}<1 & \text { if } \theta=0 \\ \alpha<\min \left\{\frac{2 b_{1}}{3 c_{2}^{2}(1+\theta)}, 2 \gamma\right\} & \text { if }-1<\theta<0 .\end{cases}
$$

Remark 2.5. Recently, Nakao [14], [15] has studied the asymptotic behavior of solutions of (E) under the similar assumptions to ours. He proved that the energy $E(t)$ satisfies a difference inequality of the form

$$
\sup _{t \leqq s \leqq t+1} E(s)^{1+\alpha} \leqq C(t+1)^{r}\{E(t)-E(t+1)\}+g(t),
$$

where $C>0, \alpha>0,1 \geqq \gamma \geqq 0$ and $g(t)$ is a non-negative function tending to zero as $t \rightarrow \infty$. From this inequality he derived the rate of the decay of $E(t)$. However, Theorems 2 and 3 give preciser results than the corresponding ones in [14], [15].

\section{$\S 3$. Proofs of Theorems}

In this section we always assume that a pair of functions $\{G, h\}$ satisfies (GH.1)-(GH.5) (in case $p+q>4$ ) or (GH.1)-(GH.3), (GH.4)' and (GH.5)' (in case $p=q=2$ ). To prove our theorems we employ the weighted energy method. We take $G(t)$ as a weight function of $E(t)$ and take $h(t)$ as an auxiliary function of $G(t)$.

\subsection{Some Lemmas}

LEMMA 3.1. 


$$
\sup _{t>t_{0}}\left\{\int_{h(t)}^{t} G^{\prime}(s)|f(s)|_{V^{*}}^{p^{\prime}} d s\right\} \equiv M_{1}<\infty,
$$

where $p^{\prime}=p /(p-1)$.

Proof. To prove this lemma, we have only to show that there exists a positive constant $M_{1}^{\prime}$ satisfying

$$
\int_{h(t)}^{t} G^{\prime}(s)|f(s)|_{W^{*}}^{p^{\prime}} d s \leqq M_{1}^{\prime}
$$

for all $t>t_{0}$ (note (2.1)).

First we shall prove (3.1) in case $p>q$. We note that

$$
\begin{aligned}
\int_{h(t)}^{t} G^{\prime}(s)|f(s)|_{W^{*}}^{p^{\prime}} d s= & \int_{h(t)}^{t}\left\{\beta(s)^{-p^{\prime} / q} G(s)^{p^{\prime} / q^{\prime}}|f(s)|_{W^{*}}^{p^{\prime}}\right\} \\
& \times\left\{\beta(s)^{p^{\prime} / q} G^{\prime}(s) G(s)^{-p^{\prime} / q^{\prime}}\right\} d s,
\end{aligned}
$$

where $q^{\prime}=q /(q-1)\left(>p^{\prime}\right)$. Using Hölder's inequality we have

$$
\begin{aligned}
&\left.\int_{h(t)}^{t} G^{\prime}(s)|f(s)|\right|_{W^{*}} ^{p^{\prime}} d s \\
& \leqq\left\{\int_{h(t)}^{t} \beta(s)^{-q^{\prime} / q} G(s)|f(s)|_{W^{*}}^{q^{\prime}} d s\right\}^{p^{\prime} / q^{\prime}} \\
& \times\left\{\int_{h(t)}^{t} \beta(s)^{p /(p-q)} G^{\prime}(s)^{q(p-1) /(p-q)} G(s)^{-p(q-1) /(p-q)} d s\right\}^{1-p^{\prime} / q^{\prime}},
\end{aligned}
$$

which, together with (GH.3) and (GH.4) (iii), implies (3.1).

In case $p \leqq q$, we have

$$
\begin{aligned}
\left.\int_{h(t)}^{t} G^{\prime}(s)|f(s)|\right|_{W^{*}} ^{p^{\prime}} d s= & \int_{h(t)}^{t}\left\{\left.\beta(s)^{-\iota^{\prime} / q} G(s)|f(s)|\right|_{W^{*}} ^{q^{\prime}}\right\} \\
& \times\left.\left\{\beta(s)^{q^{\prime} / q} G^{\prime}(s) G(s)^{-1}\right\}|f(s)|\right|_{W^{\prime}} ^{p^{\prime}} d s \\
\leqq & \left.\left|\beta^{1 /(q-1)} \cdot G^{\prime} \cdot G^{-1}\right|_{L^{\infty}\left(h\left(t_{0}\right), \infty\right)} \cdot|f|\right|_{L^{\infty}\left(0, q^{\prime}, W^{*}\right)} ^{p^{\prime}} \\
& \times\left.\int_{h(t)}^{t} \beta(s)^{-q^{\prime} / q} G(s)|f(s)|\right|_{W^{*}} ^{q^{\prime}} d s .
\end{aligned}
$$

Hence, recalling (A.3), (GH.3), (GH.4) and $(\mathrm{GH} \cdot 4)^{\prime}$, we see that (3.1) holds.

[Q.E.D.]

LEMMA 3.2.

$$
\int_{0}^{\infty} \beta(s)^{-q^{\prime} / q}|f(s)|_{W^{*}}^{q^{\prime}} d s \equiv M_{2}<\infty
$$

where $q^{\prime}=q /(q-1)$. 
Proof. To prove this lemma, we define functions $h_{i}(t)(i=0,1,2$, ..) as follows:

$$
\begin{aligned}
& h_{0}(t)=t \quad \text { and } \quad h_{i}(t)=h\left(h_{i-1}(t)\right) \quad \text { for } t \text { such that } h_{i-1}(t)>t_{0} \text {, } \\
& =h\left(t_{0}\right) \quad \text { for } t \text { such that } h_{i-1}(t) \leqq t_{0} .
\end{aligned}
$$

Now let $T$ and $T^{\prime}$ be arbitrary numbers such that $T^{\prime}>T>t_{0}$ and fix them. Then, by (GH.2), there exists a positive integer $n$ such that

$$
h_{n-1}\left(T^{\prime}\right)>T \text { and } h_{n}\left(T^{\prime}\right) \leqq T .
$$

Since the function $t \in\left[T^{\prime}, \infty\right) \mapsto h_{n}(t)$ is continuous and strictly monotone increasing, we can take a number $T^{\prime \prime}\left(\geqq T^{\prime}\right)$ such that $h_{n}\left(T^{\prime \prime}\right)=T$ (note that $\left.\lim _{t \rightarrow \infty} h_{n}(t)=\infty\right)$. Using (GH.1)-(GH.3), we have with the aid of such functions $\left\{h_{i}\right\}$

$$
\begin{aligned}
\int_{T}^{T^{\prime}} \beta(s)^{-q^{\prime} / q}|f(s)|_{W^{*}}^{q^{\prime}} d s & \leqq \int_{T}^{T^{\prime \prime}} \beta(s)^{-q^{\prime} / q}|f(s)|_{W^{*}}^{q^{\prime}} d s \\
& =\sum_{i=1}^{n} \int_{h_{i}\left(T^{\prime \prime \prime}\right)}^{h_{i-1}\left(T^{\prime \prime}\right)} \beta(s)^{-q^{\prime} / q}|f(s)|_{W^{*}}^{q^{\prime}} d s \\
& \leqq L^{q^{\prime}} \sum_{i=1}^{n} G\left(h_{i}\left(T^{\prime \prime}\right)\right)^{-1} .
\end{aligned}
$$

On the other hand, (GH.5) (or (GH.5)') implies

$$
\sup _{t>t_{0}} \frac{G(h(t))}{G(t)} \equiv d<1 \text {. }
$$

Therefore,

$$
G\left(h_{i}\left(T^{\prime \prime}\right)\right)^{-1} \leqq d^{n-i} G(T)^{-1} \quad \text { for } 1 \leqq i \leqq n .
$$

Combining (3.3) and (3.4) we get

$$
\int_{T}^{T^{\prime}} \beta(s)^{-q^{\prime} / q}|f(s)|_{W^{\prime}}^{q^{\prime}} d s \leqq \frac{L^{q^{\prime}}}{(1-d) G(T)} .
$$

Thus we conclude the proof of Lemma 3.2 because $T$ and $T^{\prime}\left(T^{\prime}>T\right.$ $>t_{0}$ ) are arbitrary and $G(T)$ tends to $\infty$ as $T \rightarrow \infty$.

[Q.E.D.]

LEMMa 3.3. Let $u$ be a solution of (E) with the weak conservation property. Then

$$
\sup _{t \geq 0} E(t) \equiv M_{3}<\infty
$$


where $E(t)$ is the energy defined by (2.5).

Proof. We note that $u$ satisfies (2.6). Putting $\psi=1$ in (2.6) we have

$$
\begin{aligned}
& E(t)+\int_{s}^{t}\left(B(r) u^{\prime}(r), u^{\prime}(r)\right)_{W} d r \\
& \leqq E(s)+\int_{s}^{t}|f(r)|_{W *} \cdot\left|u^{\prime}(r)\right|_{W} d r \quad \text { for } 0 \leqq s \leqq t,
\end{aligned}
$$

from which we obtain, by (A.2) and Hölder's inequality,

$$
\begin{aligned}
E(t)+ & b_{1} \int_{s}^{t} \beta(r)\left|u^{\prime}(r)\right|_{W}^{q} d r \\
& \leqq E(s)+\left\{\int_{s}^{t} \beta(r)\left|u^{\prime}(r)\right|_{W}^{q} d s\right\}^{1 / q}\left\{\int_{s}^{t} \beta(r)^{-q^{\prime} / q}|f(r)|_{W^{*}}^{q^{\prime}} d r\right\}^{1 / q^{\prime}}
\end{aligned}
$$

for $0 \leqq s \leqq t$, where $q^{\prime}=q /(q-1)$. Therefore, using Young's inequality and Lemma 3.2, we see that $\sup _{t \geqq 0} E(t)<\infty$.

Finally we shall prove the following lemma which plays an important role in the proof of Theorem 1.

LEMMA 3.4. Let $u$ be a solution of (E) with the weak conservation property. Then there exist positive constants $N_{1}, N_{2}, N_{3}$ and $T$ $\left(\geqq t_{0}\right)$ satisfying

$$
\begin{aligned}
& N_{1}>1+2 N_{2}, \\
& \begin{aligned}
N_{1} \int_{h(t)}^{t} G^{\prime}(s) E(s) d s \leqq & \int_{h(t)}^{t} G(s)\left(B(s) u^{\prime}(s), u^{\prime}(s)\right)_{W} d s \\
& +N_{2}\{G(t) E(t)+G(h(t)) E(h(t))\}+N_{3} \quad \text { for } t>T,
\end{aligned}
\end{aligned}
$$

and

$$
\sup \frac{G(h(t))}{G(t)}<1-\frac{2 N_{2}}{N_{1}-1}
$$

Proof. Since $u$ satisfies (2.4), we have, by putting $\phi=G^{\prime} u$ in (2.4),

$$
\begin{aligned}
\int_{r}^{t} G^{\prime}(s)(A u(s), u(s))_{V} d s= & \int_{r}^{t}\left\{G^{\prime \prime}(s)\left(u^{\prime}(s), u(s)\right)_{H}+G^{\prime}(s)\left|u^{\prime}(s)\right|_{H}^{2}\right. \\
& \left.+G^{\prime}(s)(f(s), u(s))_{V}-G^{\prime}(s)\left(B(s) u^{\prime}(s), u(s)\right)_{W}\right\} d s \\
& -G^{\prime}(t)\left(u^{\prime}(t), u(t)\right)_{H}+G^{\prime}(r)\left(u^{\prime}(r), u(r)\right)_{H}
\end{aligned}
$$

for every $0 \leqq r \leqq t$. On the other hand, by (2.3), 


$$
\int_{r}^{t} G^{\prime}(s) E(s) d s \leqq \int_{r}^{t} G^{\prime}(s)\left\{(A u(s), u(s))_{V}+\frac{1}{2}\left|u^{\prime}(s)\right|_{H}^{2}\right\} d s \quad \text { for } 0 \leqq r \leqq t .
$$

Consequently, we get

$$
\begin{aligned}
\int_{h(t)}^{t} G^{\prime}(s) E(s) d s \leqq & \int_{h(t)}^{t} G^{\prime \prime}(s)\left(u^{\prime}(s), u(s)\right)_{H} d s+\frac{3}{2} \int_{h(t)}^{t} G^{\prime}(s)\left|u \iota^{\prime}(s)\right|_{H}^{2} d s \\
& +\int_{h(t)}^{t}\left\{-G^{\prime}(s)\left(B(s) u^{\prime}(s), u(s)\right)_{W}\right\} d s \\
& +\int_{h(t)}^{t} G^{\prime}(s)(f(s), u(s))_{V} d s \\
& +\left\{-G^{\prime}(t)\left(u^{\prime}(t), u(t)\right)_{H}+G^{\prime}(h(t))\left(u^{\prime}(h(t)), u(h(t))\right)_{H}\right\} \\
& \equiv I_{1}(t)+I_{2}(t)+I_{3}(t)+I_{4}(t)+I_{5}(t)
\end{aligned}
$$

for $t>t_{0}$.

Now we shall estimate each $I_{i}(t)(i=1, \ldots, 5)$ in terms of $\left|G^{\prime} E\right|_{L^{1}(h(t), t)},\left|G\left(B(\cdot) u^{\prime}, u^{\prime}\right)_{W}\right|_{L^{1}(h(t), t)}, G(t) E(t)$ and $G(h(t)) E(h(t))$. By virtue of (2.1), (2.2) and (A.1) we have

$$
\begin{aligned}
I_{1}(t)= & \int_{h(t)}^{t} G^{\prime \prime}(s)\left(u^{\prime}(s), u(s)\right)_{H} d s \\
\leqq & \int_{h(t)}^{t}\left|G^{\prime \prime}(s)\right| \cdot\left|u^{\prime}(s)\right|_{H} \cdot|u(s)|_{H} d s \\
\leqq & c_{1} c_{2} \int_{h(t)}^{t}\left|G^{\prime \prime}(s)\right| \cdot\left|u^{\prime}(s)\right|_{H} \cdot|u(s)|_{V} d s \\
\leqq & \frac{\sqrt{2} c_{1} c_{2}}{a^{1 / p}} \int_{h(t)}^{t}\left\{\frac{1}{2} G^{\prime}(s)\left|u^{\prime}(s)\right|_{H}^{2}\right\}^{1 / 2} \cdot\left\{G^{\prime}(s) F_{A}(u(s))\right\}^{1 / p} \\
& \times\left\{\left|G^{\prime \prime}(s)\right| G^{\prime}(s)^{-(p+2) /(2 p)}\right\} d s,
\end{aligned}
$$

from which it follows that

$$
I_{1}(t) \leqq\left.\left. 2 k_{1}(p) \cdot\left|\frac{1}{2} G^{\prime}\right| u^{\prime}\right|_{H} ^{2}\right|_{L^{1}(h(t), t)} ^{1 / 2} \cdot\left|G^{\prime} F_{A}(u)\right|_{L^{1}(h(t), t)}^{1 / p},
$$

where we have used Hölder's inequality.

$I_{2}(t)$ can be estimated in the same way as above

$$
\begin{aligned}
I_{2}(t) & =\frac{3}{2} \int_{h(t)}^{t} G^{\prime}(s)\left|u^{\prime}(s)\right|_{H}^{2} d s \\
& \leqq \frac{3 c_{2}^{2}}{2 b_{1}^{2 / q}} \int_{h(t)}^{t}\left\{G(s)\left(B(s) u^{\prime}(s), u^{\prime}(s)\right)_{W}\right\}^{2 / q} \cdot\left\{G^{\prime}(s)(\beta(s) G(s))^{-2 / q}\right\} d s ;
\end{aligned}
$$

so that 


$$
I_{2}(t) \leqq k_{3}(q) \cdot \mid G\left(B(\cdot) u^{\prime}, u^{\prime}\right)_{W}^{\mid L^{1}(h(t), t)} \cdot
$$

To estimate $I_{3}(t)$ we devide the proof into two cases: $p \geqq q$ and $p<q$. When $p \geqq q$, we see by (2.1), (A.1) and (A.2) that

$$
\begin{aligned}
I_{3}(t)= & \int_{h(t)}^{t}\left\{-G^{\prime}(s)\left(B(s) u^{\prime}(s), u(s)\right)_{W}\right\} d s \\
\leqq & \frac{b_{2} c_{1}}{a^{1 / p} b_{1}^{(q-1) / q}} \int_{h(t)}^{t}\left\{G(s)\left(B(s) u^{\prime}(s), u^{\prime}(s)\right)_{W}\right\}^{(q-1) / q} \\
& \times\left\{G^{\prime}(s) F_{A}(u(s))\right\}^{1 / p} \cdot\left\{\beta(s)^{1 / q} G^{\prime}(s)^{(p-1) / p} G(s)^{-(q-1) / q}\right\} d s .
\end{aligned}
$$

Hence, using Hölder's inequality again, we have

$$
I_{3}(t) \leqq k_{4}(p, q) \cdot\left|G\left(B(\cdot) u^{\prime}, u^{\prime}\right)_{W}\right|_{L^{1}(h(t), t)}^{(q-1) / q} \cdot\left|G^{\prime} F_{A}(u)\right|_{L^{1}(h(t), t)}^{\mid p}
$$

for $p \geqq q$. When $p<q$, we get

$$
\begin{aligned}
I_{3}(t) \leqq & \frac{b_{2} c_{1}}{a^{1 / q} b_{1}^{(q-1) / q}} \int_{h(t)}^{t}\left\{G(s)\left(B(s) u^{\prime}(s), u^{\prime}(s)\right)_{W}\right\}^{(q-1) / q} \cdot\left\{G^{\prime}(s) F_{A}(u(s))\right\}^{1 / q} \\
& \times\left\{\beta(s) G^{\prime}(s)^{q-1} G(s)^{-(q-1)}\right\}^{1 / q} \cdot|u(s)|_{V}^{(q-p) / q} d s .
\end{aligned}
$$

Therefore, using Hölder's inequality, we find that for $p<q$

$$
\begin{aligned}
I_{3}(t) \leqq & \frac{b_{2} c_{1} M_{3}^{(q-p) / p q}}{a^{1 / p} b_{1}^{(q-1) / q}} \cdot \sup _{h(t) \leqq s \leqq t}\left\{\frac{\beta(s) G^{\prime}(s)^{q-1}}{G(s)^{q-1}}\right\}^{1 / q} \\
& \times\left|G\left(B(\cdot) u^{\prime}, u^{\prime}\right)_{W}\right|_{L^{1}(h(t), t)}^{(q-1) / q} \cdot\left|G^{\prime} F_{A}(u)\right|_{L^{1}(h(t), t)}^{1 / q},
\end{aligned}
$$

where $M_{3}$ is the positive constant in Lemma 3.3.

$I_{4}(t)$ is estimated as follows:

$$
\begin{aligned}
I_{4}(t) & =\int_{h(t)}^{t} G^{\prime}(s)(f(s), u(s))_{V} d s \\
& \leqq a^{-1 / p} M_{1}^{(p-1) / p}\left|G^{\prime} F_{A}(u)\right|_{L^{1}(h(t), t)}^{1 / p},
\end{aligned}
$$

where $M_{1}$ is the constant in Lemma 3.1.

Finally we note that

$$
\begin{aligned}
\left|G^{\prime}(s)\left(u^{\prime}(s), u(s)\right)_{H}\right| \leqq & \frac{\sqrt{2} c_{1} c_{2}}{a^{1 / p}}\left\{\frac{1}{2} G(s)\left|u^{\prime}(s)\right|_{H}^{2}\right\}^{1 / 2} \cdot\left\{G(s) F_{A}(u(s))\right\}^{1 / p} \\
& \times\left\{G^{\prime}(s) G(s)^{-(p+2) /(2 p)}\right\}
\end{aligned}
$$

holds for every $s \geqq 0$ (by (2.1), (2.2) and (A.1)). Thus 


$$
\begin{aligned}
I_{5}(t)= & \left\{-G^{\prime}(t)\left(u^{\prime}(t), u(t)\right)_{H}+G^{\prime}(h(t))\left(u^{\prime}(h(t)), u(h(t))\right)_{H}\right\} \\
\leqq & k_{2}(p)\left[\left\{\frac{1}{2} G(t)\left|u^{\prime}(t)\right|_{H}^{2}\right\}^{1 / 2} \cdot\left\{G(t) F_{A}(u(t))\right\}^{1 / p}\right. \\
& \left.+\left\{\frac{1}{2} G(h(t))\left|u^{\prime}(h(t))\right|_{H}^{2}\right\}^{1 / 2} \cdot\left\{G(h(t)) F_{A}(u(h(t)))\right\}^{1 / p}\right]
\end{aligned}
$$

Then, by using (GH.4), (GH.5) (or (GH.4)', (GH.5)') and Young's inequality, we deduce from (3.6)-(3.11) that there exist positive constants $C_{i}(i=1,2,3,4)$ and $T\left(\geqq t_{0}\right)$ satisfying

$$
C_{1}+C_{2}+2 C_{3}<1 \text {, }
$$

$$
\begin{aligned}
\int_{h(t)}^{t} G^{\prime}(s) E(s) d s \leqq & C_{1} \int_{h(t)}^{t} G^{\prime}(s) E(s) d s \\
& +C_{2} \int_{h(t)}^{t} G(s)\left(B(s) u^{\prime}(s), u^{\prime}(s)\right)_{W} d s \\
& +C_{3}\{G(t) E(t)+G(h(t)) E(h(t))\}+C_{4}, \\
& \text { for } t>T,
\end{aligned}
$$

and

$$
\sup _{t>T^{\prime}} \frac{G(h(t))}{G(t)}<1-\frac{2 C_{3}}{1-C_{1}-C_{2}} .
$$

Consequently, Lemma 3.4 follows from (3.12)-(3.14) with $N_{1}=\left(1-\mathrm{C}_{1}\right) / C_{2}$, $N_{2}=C_{3} / C_{2}$ and $N_{3}=C_{4} / C_{2}$.

[Q.E.D.]

Remark 3.1. Suppose that $A$ satisfies (A.1)' in place of (A.1) (see Remark 2.2); Then there exists a positive number $c_{3}$ satisfying

$$
|v|_{W} \leqq c_{3}|v|_{V_{1}} \quad \text { for } v \in V_{1} \text {, }
$$

where $V_{1}$ is a real Banach space such that $V \subset V_{1} \subset W$. Furthermore, suppose that we can take a pair $\{G, h\}$ satisfying (GH.1)-(GH.5) (in case $p+q>4$ ) or (GH.1)-(GH.3), (GH.4) $)^{\prime}$ and (GH.5)' (in case $p=q=2$ ) with $\alpha$ and $c_{1}$ replaced by $\alpha_{1}$ and $c_{3}$ respectively. Then we can show (3.7)-(3.11) quite in the same way as in the proof of Lemma 3.4; so that the conclusion of Lemma 3.4 remains true.

In this case, Theorem 1 also holds true (see the proof of Theorem 1).

\subsection{Proof of Theorem 1}

We put

$$
\sup _{t>T} \frac{G(h(t))}{G(t)}=d
$$


Since, by Lemma 3.4,

$$
d<1-\frac{2 N_{2}}{N_{1}-1} \text { and } 1+2 N_{2}<N_{1}
$$

we can take a positive number $\varepsilon_{0}$ such that

$$
\varepsilon_{0}<1-\frac{1-d}{(1-d) N_{1}-2 N_{2}} .
$$

Now let $u$ be a solution of (E) with the weak conservation property. Then, setting $\psi=G$ and $s=h(t)$ in (2.6) gives

$$
\begin{aligned}
& G(t) E(t)+\int_{h(t)}^{t} G(s)\left(B(s) u^{\prime}(s), u^{\prime}(s)\right)_{W} d s \\
& \quad \leqq G(h(t)) E(h(t))+\int_{h(t)}^{t} G^{\prime}(s) E(s) d s+\int_{h(t)}^{t} G(s)\left(f(s), u^{\prime}(s)\right)_{W} d s
\end{aligned}
$$

for $t>t_{0}$. On the other hand, by (A.2) we have

$$
\begin{aligned}
\int_{h(t)}^{t} G(s)\left(f(s), u^{\prime}(s)\right)_{W} d s \leqq & b_{1}^{-1 / q} \int_{h(t)}^{t}\left\{G(s)\left(B(s) u^{\prime}(s), u^{\prime}(s)\right)_{W}\right\}^{1 / q} \\
& \times\left\{\beta(s)^{-1 / q} G(s)^{(q-1) / q}|f(s)|_{W^{*}}\right\} d s
\end{aligned}
$$

so we find, by recalling (GH.3), that the right-hand side of the above inequality is bounded by

$$
\varepsilon_{0} \int_{h(t)}^{t} G(s)\left(B(s) u^{\prime}(s), u^{\prime}(s)\right)_{W} d s+C_{5} .
$$

Here $\varepsilon_{0}$ is a positive constant satisfying (3.16) and $C_{5}$ is some positive constant. Hence it follows from (3.17) that

$$
\begin{array}{r}
G(t) E(t)+\left(1-\varepsilon_{0}\right) \int_{h(t)}^{t} G(s)\left(B(s) u^{\prime}(s), u^{\prime}(s)\right)_{W} d s \\
\leqq G(h(t)) E(h(t))+\int_{h(t)}^{t} G^{\prime}(s) E(s) d s+C_{5}
\end{array}
$$

holds for every $t>t_{0}$.

We shall combine (3.18) with the second inequality in Lemma 3.4; then

$$
\begin{array}{r}
\left\{1-N_{2}\left(1-\varepsilon_{0}\right)\right\} G(t) E(t)+\left\{N_{1}\left(1-\varepsilon_{0}\right)-1\right\} \int_{h(t)}^{t} G^{\prime}(s) E(s) d s \\
\leqq\left\{1+N_{2}\left(1-\varepsilon_{0}\right)\right\} G(h(t)) E(h(t))+N_{3}\left(1-\varepsilon_{0}\right)+C_{5} \\
\text { for } t>T
\end{array}
$$


But the function

$$
t \mapsto E(t)-C_{6} \int_{0}^{t} \beta(r)^{-q^{\prime} / q} \cdot|f(r)|_{W^{*}}^{q^{\prime}} d r, \quad q^{\prime}=\frac{q}{q-1},
$$

is monotone non-increasing for some $C_{6}>0$ (note (3.5)) ; so that

$$
\begin{aligned}
\int_{h(t)}^{t} G^{\prime}(s) E(s) d s & \geqq \int_{h(t)}^{t} G^{\prime}(s)\left\{E(t)-C_{6} \int_{s}^{t} \beta(r)^{-q^{\prime} / q}|f(r)|_{W^{\prime}}^{\prime} d r\right\} d s \\
& \geqq(1-d) G(t) E(t)-C_{6} L^{q^{\prime}} \quad \text { for } t>T,
\end{aligned}
$$

where we have used (3.15) and (GH.3). Therefore, (3.19) and (3.20) imply that for some $C_{7}$

$$
\begin{aligned}
{\left[\left(1-\varepsilon_{0}\right)\right.} & \left.\left\{N_{1}(1-d)-N_{2}\right\}+d\right] G(t) E(t) \\
& \leqq\left\{1+N_{2}\left(1-\varepsilon_{0}\right)\right\} G(h(t)) E(h(t))+C_{7}, \quad t>T,
\end{aligned}
$$

because $N_{1}\left(1-\varepsilon_{0}\right)-1>0$ by (3.16). Hence, by virtue of (3.16) we see that there exist some constants $0<\alpha<1$ and $C_{8}>0$ satisfying

$$
G(t) E(t) \leqq \alpha G(h(t)) E(h(t))+C_{8} \quad \text { for } t>T .
$$

Finally to conclude the proof of this theorem, we take functions $\left\{h_{i}(t)\right\}$ defined by (3.2). We recall that, for each $t>T$, there exists a positive integer $n$ such that $h_{n-1}(t)>T$ and $h_{n}(t) \leqq T$. Then it follows from (3.21) that

$$
\begin{aligned}
G(t) E(t) & \leqq \alpha^{n} G\left(h_{n}(t)\right) E\left(h_{n}(t)\right)+C_{8} \sum_{i=0}^{n-1} \alpha^{i} \\
& \leqq \sup _{0 \leqq s \leqq T}\{G(s) E(s)\}+\frac{C_{8}}{1-\alpha},
\end{aligned}
$$

which completes the proof.

[Q.E.D.]

Remark 3.2. Let $p<q$. If it is known that $E(t)$ decays to zero as $t \rightarrow \infty$, we can prove Theorem 1 with (GH.4) (iii) replaced by the following weaker condition

$$
\left|\beta \cdot\left(G^{\prime}\right)^{q-1} \cdot G^{-(q-1)}\right|_{L^{\infty}\left(h\left(t_{0}\right), \infty\right)}<\infty .
$$

In fact, since $E(t) \rightarrow 0$ as $t \rightarrow \infty$, the constant $M_{3}$ in (3.9)' can be taken sufficiently small for $t$ large enough; so that for any $\varepsilon>0$ there exists a sufficiently large $T$ such that 


$$
I_{3}(t) \leqq \varepsilon\left\{\left|G\left(B(\cdot) u^{\prime}, u^{\prime}\right)_{W}\right|_{L^{1}(h(t), t)}+\left|G^{\prime} F_{A}(u)\right|_{L_{1}(h(t), t)}\right\}
$$

holds for all $t>T$. Hence we can show that not only Lemma 3.4 but also Theorem 1 holds true.

Remark 3.3. Even if the operator $A: V \rightarrow V^{*}$ depends on $t$, we can employ the method used here to investigate the rate of the dacay of solutions of (E). However, conditions on $\{G, h\}$ will become more complicate in this case (cf. Nakao [15]).

\subsection{Proof of Theorem 2}

Let $\beta(t)=(t+1)^{\theta}$ with $-1 \leqq \theta \leqq q-1$ and let $|f(t)|_{W^{*}}=0\left(t^{-r}\right)$ with $\gamma>(q-1-\theta) / q$ as $t \rightarrow \infty$. We shall apply Theorem 1 to prove this theorem; we choose a suitable pair $\{G, h\}$ so that it satisfies (GH.1)(GH.5) (in case $p+q>4$ ) or (GH.1)-(GH.3), (GH.4)' and (GH.5)' (in case $p=q=2$ ).

(i) The case $-1<\theta<q-1$.

We put

$$
\{G(t), h(t)\}=\left\{t^{\alpha}, c t\right\}, \quad \alpha>0,0<c<1 .
$$

Let $0<c<1$ be fixed. We shall determine $\alpha$ so that conditions (GH.3) -(GH.5) (in case $p+q>4$ ) or (GH.3), (GH.4) and (GH.5)' (in case $p$ $=q=2$ ) are satisfied; clearly the pair $\{G, h\}$ defined by (3.22) satisfies (GH.1) and (GH.2).

We first treat the case $p+q>4$. From (GH.4) (i), (ii) and (iii) we have, respectively,

$$
\begin{aligned}
& \begin{cases}0<\alpha \leqq \frac{2 p}{p-2} & \text { if } p>2 \\
0<\alpha & \text { if } p=2,\end{cases} \\
& \begin{cases}0<\alpha \leqq \frac{2(1+\theta)}{q-2} & \text { if } q=2 \\
0<\alpha & \text { if } q=2,\end{cases}
\end{aligned}
$$

and

$$
\begin{cases}0<\alpha \leqq \frac{p(q-1-\theta)}{p-q} & \text { if } p>q \\ 0<\alpha & \text { if } p \leqq q\end{cases}
$$


(Take $t_{0}$ sufficiently large if necessary.) Hence (GH.4) is satisfied for every $\alpha$ such that

(3.23) $0<\alpha \leqq \delta, \quad \delta \equiv \begin{cases}\frac{2(1+\theta)}{q-2} & \text { if } p(q-2) \geqq(p-2)(1+\theta) \\ \frac{p(q-1-\theta)}{p-q} & \text { if } p(q-2)<(p-2)(1+\theta)\end{cases}$

Moreover, (GH.5) is satisfied for every $\alpha>0$ if $t_{0}$ is sufficiently large.

In case $p=q=2$, (GH.4) $)^{\prime}$ and (GH.5)' are easily verified for every $\alpha>0$ if $t_{0}$ is large enough.

Finally, it follows from (GH.3) that

$$
0<\alpha \leqq \frac{q \gamma-q+\theta+1}{q-1}
$$

Hence, all conditions on $\{G, h\}$ are verified for every $\alpha$ satisfying (3.23) and (3.24). Thus Theorem 1 assures that there exists a positive number $C$ such that

$$
E(t) \leqq C t^{-\alpha}, \quad t>0
$$

where $\alpha=\min \{\delta,(q \gamma-q+\theta+1) /(q-1\}$.

(ii) The case $\theta=q-1$.

In this case we first put

$$
\{G(t), h(t)\}=\left\{(\log (t+1))^{\rho}, \quad(t+1)^{d}-1\right\}, \quad \rho>0,1>d>0,
$$

and fix $d$. Then taking $t_{0}$ sufficiently large, we see that all conditions on $\{G, h\}$ are satisfied for every $\rho$ such that

$$
\begin{cases}0<\rho \leqq \frac{p(q-1)}{p-q} & \text { if } p>q \\ 0<\rho & \text { if } p \leqq q .\end{cases}
$$

Therefore, it follows from Theorem 1 that there exists a positive constant $C$ such that

$$
E(t) \leqq \begin{cases}C(\log (t+1))^{-p(q-1) /(p-q)} & \text { if } p>q \\ C(\log (t+1))^{-\rho} & \text { if } p \leqq q,\end{cases}
$$

where $\rho$ is an arbitrary positive number. 
In order to obtain preciser results in case $p \leqq q$, we make use of Remark 2.4, because $E(t)$ decays to zero as $t \rightarrow \infty$ (by (3.26)).

Let $p \leqq q$ and take a pair of functions $\{G, h\}$ of the form (3.22) with $c$ fixed. Then noting (3.23) and (3.24) we can determine $\alpha>0$; all conditions on $\{G, h\}$ are satisfied when

$$
\begin{cases}\alpha \leqq \mu \equiv \min \left\{\frac{2 q}{q-2}, \frac{q \gamma}{q-1}\right\} & \text { if } p<q \\ \alpha \leqq \mu \text { and } \quad \alpha<\frac{a^{1 /(p-1)} b_{1}}{\left(b_{2} c_{1}\right)^{p /(p-1)}} & \text { if } p=q .\end{cases}
$$

Consequently, it follows from Theorem 1 that for some positive constant $C$

$$
E(t) \leqq \begin{cases}C t^{-\mu}, \quad t>0, & \text { if } p<q \\ C t^{-\alpha}, \quad t>0, & \text { if } p=q,\end{cases}
$$

where $\alpha$ is a positive number satisfying

$$
\alpha \leqq \mu \text { and } \alpha<\frac{a^{1 /(p-1)} b_{1}}{\left(b_{2} c_{1}\right)^{p /(p-1)}} .
$$

Thus (3.26) and (3.27) give the desired results.

(iii) The case $\theta=-1$.

We take a pair $\{G, h\}$ of the form (3.25) and fix $0<d<1$. Then, just as in the proof of (ii), we can prove that for some $C>0$

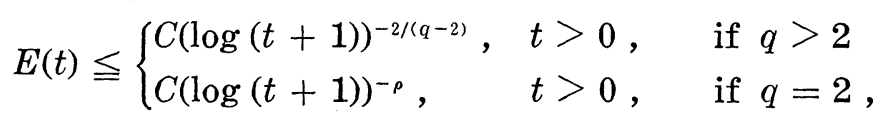

where $\rho$ is an arbitrary positive number.

In particular, when $q=2$, taking $a_{i}^{\pi}$ pair $\{G, h\}$ of the form (3.22) we can show that, for some $C>0$,

$$
E(t) \leqq C t^{-\alpha}, \quad t>0,
$$

where $\alpha$ is a positive number such that

$$
\alpha \leqq \min \left\{2(\gamma-1), \frac{2 p}{p-2}\right\} \text { and } \alpha<\frac{2 b_{1}}{3 c_{2}^{2}} \text {. }
$$

[Q.E.D.] 


\subsection{Proof of Theorem 3}

Let $p=q=2, \beta(t)=(t+1)^{\theta}$ with $-1<\theta<1$ and $|f(t)|_{W^{*}}$ $=0\left(\exp \left(-\gamma t^{1-|\theta|}\right)\right)$ with $\gamma>0$ as $t \rightarrow \infty$. We put

(3.28) $\{G(t), h(t)\}=\left\{\exp \left(\alpha t^{1-|\theta|}\right), \quad\left(t^{1-|\theta|}-c\right)^{1 /(1-|\theta|)}\right\}, \quad \alpha>0, c>0$,

and determine $\alpha$ and $c$ so that $\{G, h\}$ satisfies (GH.3), (GH.4) ${ }^{\prime}$ and (GH.5)'; (GH.1) and (GH.2) are satisfied if $t_{0}^{1-|\theta|} \geqq c$. First we fix $c>0$. Then (GH.3) is satisfied for every $\alpha>0$ such that

$$
\begin{cases}\alpha \leqq 2 \gamma & \text { if } 0 \leqq \theta<1 \\ \alpha<2 \gamma & \text { if }-1<\theta<0 .\end{cases}
$$

Moreover, by taking $t_{0}$ large enough if $\theta \neq 0$, we can verify (GH.4) for every $\alpha>0$ such that

$$
\begin{cases}\alpha<\frac{a b_{1}}{b_{2}^{2} c_{1}^{2}(1-\theta)} & \text { if } 0<\theta<1 \\ 3\left(\frac{c_{1} c_{2}}{\sqrt{2 a}}+\frac{c_{2}^{2}}{2 b_{1}}\right) \alpha+\left(\frac{b_{2} c_{1}}{\sqrt{a b_{1}}}\right) \sqrt{\alpha}<1 & \text { if } \theta=0 \\ \alpha<\frac{2 b_{1}}{2 c_{2}^{2}(1+\theta)} & \text { if }-1<\theta<0 .\end{cases}
$$

Now we fix $\alpha$ so that it satisfies (3.29) and (3.30). Then it can be shown that the pair $\{G, h\}$ defined by (3.28) satisfies (GH.5)' if $t_{0}$ (resp. $c$ ) is large enough in case $\theta \neq 0$ (resp. $\theta=0$ ). Therefore, using Theorem 1 we obtain the conclusions of Theorem 3 from (3.29) and (3.30).

[Q.E.D.]

\section{§4. Applications}

In this section we shall apply the preceding results to some nonlinear partial differential equations.

Let $\Omega$ be a bounded domain in $\boldsymbol{R}_{x}^{n}$ with smooth boundary $\Gamma$. By $W^{k, p}(\Omega)$ we mean $\left\{u ; D^{\alpha} u \in L^{p}(\Omega)\right.$ for $\left.|\alpha| \leqq k\right\}$, and by $W_{0}^{k, p}(\Omega)$ we mean the closure in $W^{k, p}(\Omega)$ of the smooth functions with compact supports in $\Omega$. In particular, for $p=2$ we write $H^{k}(\Omega)=W^{k, 2}(\Omega)$ and $H_{0}^{k}(\Omega)$ $=W_{0}^{k, 2}(\Omega)$.

\subsection{Example 1}

First we study the decay property of solutions to the nonlinear 
wave equation

$$
\begin{cases}\frac{\partial^{2} u}{\partial t^{2}}-\Delta u+(t+1)^{\theta}\left|\frac{\partial u}{\partial t}\right|^{q-2} \frac{\partial u}{\partial t}=f & \text { on } \Omega \times[0, \infty) \\ u=0 & \text { on } \Gamma \times[0, \infty),\end{cases}
$$

where $q \geqq 2$ and $\theta$ is a real number.

We take $H=L^{2}(\Omega), V=H_{0}^{1}(\Omega)$ and $W=L^{q}(\Omega)$. If we put

$$
A u=-\Delta u \text { and } B(t) v=(t+1)^{\theta}|v|^{q-2} v,
$$

then we easily see that $A: V \rightarrow V^{*}$ is the Fréchet derivative of a convex functional $F_{A}$ on $V$ defined by

$$
F_{A}(u)=\frac{1}{2} \int_{\Omega}|\operatorname{grad} u|^{2} d x \quad\left(\equiv \frac{1}{2}|u|_{V}^{2}\right)
$$

and that $B(t)$ satisfies (A.2) with $b_{1}=b_{2}=1$ and $\beta(t)=(t+1)^{\theta}$.

As for the existence of solutions of (4.1) with the initial conditions

$$
u(\cdot, 0)=u_{0} \text { and } \frac{\partial u}{\partial t}(\cdot, 0)=u_{1} \quad \text { on } \Omega,
$$

it is well known that, for each $f \in L_{\mathrm{loc}}^{2}(0, \infty ; H), u_{0} \in V$ and $u_{1} \in H$, there exists a unique solution $u$ of (4.1) and (4.2) satisfying

$$
u \in L_{\mathrm{loc}}^{\infty}(0, \infty ; V) \text { and } u^{\prime} \in L_{\mathrm{loc}}^{\infty}(0, \infty ; H) \cap L_{\mathrm{loc}}^{q}(0, \infty ; W)
$$

(see e.g. Lions [10] or Lions and Strauss [11]). Furthermore, according to the result of Strauss [17, Theorem 4.1], the solution $u$ always satisfies the energy equation

$$
E(t)+\int_{0}^{t}(s+1)^{\theta}\left|u^{\prime}(s)\right|_{W}^{q} d s=E(0)+\int_{0}^{t}\left(f(s), u^{\prime}(s)\right)_{W} d s,
$$

where the energy $E(t)$ is defined by

$$
E(t)=\frac{1}{2}\left|u^{\prime}(t)\right|_{H}^{2}+\frac{1}{2}|u(t)|_{V}^{2}
$$

Therefore, we can investigate the rate of the decay of solutions of (4.1) (see Remark 2.3).

Now suppose that $q \geqq 2$ if $n=1,2$ and $2 n /(n-2) \geqq q \geqq 2$ if $n \geqq 3$. Then, by Sobolev's lemma, there exists a positive number $s_{q}$ such that

$$
|u|_{W} \leqq s_{q}|u|_{V} \quad \text { for } u \in V
$$


so that

$$
V \subset W \subset H
$$

Hence, applying Theorems 2 and 3 to (4.1), we can obtain the following results (cf. Nakao [15] and Sattinger [16]).

THEOREM 4.1. Let $u$ be a solution of (4.1) and let $q \geqq 2$ if $n=1,2$ and $2 n /(n-2) \geqq q \geqq 2$ if $n \geqq 3$. If $-1 \leqq \theta \leqq q-1$ and $|f(t)|_{W^{*}}=O\left(t^{-r}\right)$ with $\gamma>(q-1-\theta) / q$ as $t \rightarrow \infty$, then the following decay properties (i) and (ii) hold.

(i) When $-1<\theta \leqq q-1$,

$$
E(t)=O\left(t^{-\alpha}\right) \quad \text { as } t \rightarrow \infty,
$$

where $\alpha$ is a positive number such that

$$
\begin{cases}\alpha \leqq 2 \gamma \quad \text { and } \alpha<\frac{1}{2 s_{2}^{2}} & \text { if } q=2 \text { and } \theta=1 \\ \alpha=\min \left\{\frac{2(1+\theta)}{q-2}, \frac{q \gamma-q+1+\theta}{q-1}\right\} & \text { otherwise. }\end{cases}
$$

(ii) When $\theta=-1$,

$$
E(t)= \begin{cases}O\left((\log t)^{-2 /(q-2)}\right) & \text { as } t \rightarrow \infty \text { if } q>2 \\ O\left(t^{-\alpha}\right) & \text { as } t \rightarrow \infty \text { if } q=2,\end{cases}
$$

where $\alpha$ is a positive number satisfying $\alpha<2 \gamma-2$ and $\alpha<2 / 3$.

In particular, if $p=q=2,-1<\theta<1$ and $|f(t)|_{W^{*}}=O\left(\exp \left(-\gamma t^{1-|\theta|}\right)\right)$ with $\gamma>0$ as $t \rightarrow \infty$, then for some sufficiently small $\alpha>0$

$$
E(t)=O\left(\exp \left(-\alpha t^{1-|\theta|}\right)\right) \quad \text { as } t \rightarrow \infty .
$$

Remark 4.1. When $\theta=0$ and $f(x, t)$ tends to a function $F(x)$ as $t \rightarrow \infty$, Nakao [12] has investigated the rate of the convergence of $u(x, t)$ to the corresponding steady state solution $\bar{u}(x)$ (see also [13]). Our method can also apply to the study of the stability of solutions of (4.1).

Remark 4.2. Levine and Murray [9] has discussed the asymptotic behavior for solutions to semilinear wave equations and obtained their lower bounds. In particular, when $q=2, \theta=-1$ and $f \equiv 0$ in (4.1) (Euler-Poisson-Darboux equation), they showed that solutions of (4.1) satisfy, for all $t$ sufficiently large and some $C>0$ and $\rho>0$, 


$$
E(t) \geqq C t^{-\rho},
$$

unless $u \equiv 0$ (cf. Theorem 4.1 (ii)).

\subsection{Example 2}

We consider the nonlinear equation of the form

$$
\begin{cases}\frac{\partial^{2} u}{\partial t^{2}}-\sum_{i=1}^{n} \frac{\partial}{\partial x_{i}}\left(\left|\frac{\partial u}{\partial x_{i}}\right|^{p-2} \frac{\partial u}{\partial x_{i}}\right)-(t+1)^{\theta} \Delta \frac{\partial u}{\partial t}=f & \text { on } \Omega \times[0, \infty), \\ u=0 & \text { on } \Gamma \times[0, \infty),\end{cases}
$$

where $p \geqq 2$ and $\theta \leqq 0$.

Take $H=L^{2}(\Omega), V=W_{0}^{1, p}(\Omega)$ and $W=H_{0}^{1}(\Omega)$. If we put

$$
A u=-\sum_{i=1}^{n} \frac{\partial}{\partial x_{i}}\left(\left|\frac{\partial u}{\partial x_{i}}\right|^{p-2} \frac{\partial u}{\partial x_{i}}\right)
$$

and

$$
B(t) v=-(t+1)^{\theta} \Delta v
$$

we easily see that $A: V \rightarrow V^{*}$ is the Fréchet derivative of a convex functional $F_{A}$ defined by

$$
F_{A}(u)=\frac{1}{p} \int_{\Omega} \sum_{i=1}^{n}\left|\frac{\partial u}{\partial x_{i}}\right|^{p} d x \quad\left(\equiv \frac{1}{p}|u|_{V}^{p}\right)
$$

and $B(t): W \rightarrow W^{*}$ satisfies (A.2) with $b_{1}=b_{2}=1, q=2$ and $\beta(t)=(t+1)^{\theta}$.

When $\theta=0$, Tsutsumi [18] treated the initial boundary value problem for (4.3) with initial conditions

$$
u(\cdot, 0)=u_{0} \text { and } \frac{\partial u}{\partial t}(\cdot, 0)=u_{1} \quad \text { on } \Omega,
$$

and showed that, for each $f \in L_{\mathrm{loc}}^{2}(0, \infty ; H), u_{0} \in V$ and $u_{1} \in H$, there exists a solution $u$ of (4.3) and (4.4) satisfying

$$
u \in L_{\mathrm{loc}}^{\infty}(0, \infty ; V) \text { and } u^{\prime} \in L_{\mathrm{loc}}^{\infty}(0, \infty ; H) \cap L_{\mathrm{loc}}^{2}(0, \infty ; W) \text {, }
$$

(for the special case $n=1$ and $\theta=0$, see e.g. Caughey and Ellison [6], Greenberg [7] and Greenberg, MacCamy and Mizel [8]). He employed the Galerkin's method to construct such a solution $u$ of (4.3) and (4.4).

When $\theta \leqq 0$, we can apply Tsutsumi's technics to the initial boundary 
value problem (4.3) and (4.4); so that, for each $f \in L_{\mathrm{loc}}^{2}(0, \infty ; H), u_{0} \in V$ and $u_{1} \in H$, there exists a solution $u$ of (4.3) and (4.4) satisfying (4.5). Moreover, such $u$ has the weak conservation property: for every $0 \leqq s$ $\leqq t$ and every positive $C^{1}$-function $\psi$,

$$
\begin{aligned}
& \psi(t) E(t)+\int_{s}^{t} \psi(r)(r+1)^{\theta}\left|u^{\prime}(r)\right|_{W}^{2} d r \\
& \leqq \psi(s) E(s)+\int_{s}^{t} \psi^{\prime}(r) E(r) d r+\int_{s}^{t} \psi(r)\left(f(r), u^{\prime}(r)\right)_{W} d r,
\end{aligned}
$$

where $E(t)=\frac{1}{2}\left|u^{\prime}(t)\right|_{H}^{2}+F_{A}(u(t))$. We shall prove these facts briefly.

Let $\left\{u_{m}\right\}$ be a sequence of approximate solutions to (4.3) and (4.4) defined by the Galerkin's method (for details, see Tsutsumi [18]). Then, almost in the same procedure as in [18], we can choose a convergent subsequence $\left\{u_{\mu}\right\}$ of $\left\{u_{m}\right\}$ such that: weakly star in $L^{\infty}(0, T ; V)$ and weakly in $L^{p}(0, T ; V)$ for every $T>0$;

(4.8) $\quad u_{\mu}^{\prime} \rightarrow u^{\prime}$ weakly star in $L^{\infty}(0, T ; H)$, weakly in $L^{2}(0, T ; W)$ and strongly in $L^{2}(0, T ; H)$ for every $T>0$;

where $u$ is a solution of (4.3) and (4.4). On the other hand, by the monotonicity of $A$, we have

$$
\begin{aligned}
& \lim _{\mu \rightarrow \infty} \int_{0}^{T}\left(A u_{\mu}(s), u_{\mu}(s)\right)_{V} d s=\lim _{\mu \rightarrow \infty} \int_{0}^{T}\left|u_{\mu}(s)\right|_{V}^{p} d s \\
&=\int_{0}^{T}(A u(s), u(s))_{V} d s=\int_{0}^{T}|u(s)|_{V}^{p} d s \quad \text { for every } T \geqq 0,
\end{aligned}
$$

(cf. [18, (2.27) and (2.28)]), which, together with (4.7), implies

$$
u_{\mu} \rightarrow u \quad \text { strongly in } L^{p}(0, T ; V) \text { for every } T>0 \text {, }
$$

because $L^{p}(0, T ; V)$ is a uniformly convex Banach space. Now we recall that the approximate function $u_{\mu}$ satisfies, for every $0 \leqq s \leqq t$ and every positive $C^{1}$-function $\psi$,

$$
\begin{aligned}
& \psi(t) E_{\mu}(t)+\int_{s}^{t} \psi(r)(r+1)^{\theta}\left|u_{\mu}^{\prime}(r)\right|_{W}^{2} d r \\
& =\psi(s) E_{\mu}(s)+\int_{s}^{t} \psi^{\prime}(r) E_{\mu}(r) d r+\int_{s}^{t} \psi(r)\left(f(r), u_{\mu}^{\prime}(r)\right)_{W} d r,
\end{aligned}
$$


where $E_{\mu}(t)=\frac{1}{2}\left|\mu_{\mu}^{\prime}(t)\right|_{H}^{2}+F_{A}\left(u_{\mu}(t)\right)$. Hence it follows from (4.8) and (4.9) that (4.6) holds for almost every $s$ and $t(0 \leqq s \leqq t)$. Therefore, we may conclude that $u$, after redefinition on a set of measure zero, satisfies (4.6) for every $0 \leqq s \leqq t$. (Note that the function

$$
t \mapsto E(t)-c \int_{0}^{t}|f(s)|_{W^{*}}^{2} d s
$$

is monotone non-increasing for some $c>0$.)

Thus we have shown the existence of a solution $u$ of (4.3) which satisfies (4.6). Hence, applying Theorems 2 and 3 to the study of the asymptotic behavior of such $u$, we have

THEOREM 4.2. Let $-1 \leqq \theta \leqq 0$ and let $u$ be a solution of (4.3) satisfying (4.6). If $|f(t)|_{W^{*}}=O\left(t^{-r}\right)$ with $\gamma>(1-\theta) / 2$ as $t \rightarrow \infty$, then

$$
E(t)=O\left(t^{-\alpha}\right) \quad \text { as } t \rightarrow \infty,
$$

where $\alpha$ is a positive number satisfying

$$
\begin{cases}\alpha=\min \left\{\frac{p(1-\theta)}{p-2}, 2 \gamma-1+\theta\right\} & \text { if }-1<\theta \leqq 0, \\ \alpha \leqq \min \left\{\frac{2 p}{p-2}, 2 \gamma-2\right\} \quad \text { and } \quad \alpha<\frac{2}{3 s_{2}^{2}} \quad \text { if } \theta=-1 .\end{cases}
$$

In particular, if $p=2,-1<\theta \leqq 0$ and $|f(t)|_{W^{*}}=O\left(\exp \left(-\gamma t^{1+\theta}\right)\right)$ with $\gamma>0$ as $t \rightarrow \infty$, then for some sufficiently small $\alpha>0$,

$$
E(t)=O\left(\exp \left(-\alpha t^{1+\theta}\right)\right) \quad \text { as } t \rightarrow \infty .
$$

\subsection{Example 3}

In this subsection we denote by $\Omega$ an open interval $(0,1)$. We discuss the rate of the decay of solutions to the nonlinear beam equation

$$
\left\{\begin{array}{lr}
\frac{\partial^{2} u}{\partial t^{2}}+\frac{\partial^{4} u}{\partial x^{4}}-\left(a+\int_{\Omega}\left|\frac{\partial u}{\partial y}(y, t)\right|^{2} d y\right) \frac{\partial^{2} u}{\partial x^{2}}+(t+1)^{\theta} \frac{\partial u}{\partial t}=f \\
u=\frac{\partial^{2} u}{\partial x^{2}}=0 & \text { on } \Omega \times[0, \infty), \\
\text { on } \Gamma \times[0, \infty),
\end{array}\right.
$$


where $a$ and $\theta$ are real numbers; the boundary conditions in (4.10) correspond to the case when the ends of the beam are hinged.

We take $H=W=L^{2}(\Omega)$ and $V=H^{2}(\Omega) \cap H_{0}^{1}(\Omega)$. Put

$$
(A u, v)_{V}=\int_{\Omega} \frac{\partial^{2} u}{\partial x^{2}} \cdot \frac{\partial^{2} v}{\partial x^{2}} d x+\left(a+\int_{\Omega}\left|\frac{\partial u}{\partial x}\right|^{2} d x\right) \int_{\Omega} \frac{\partial u}{\partial x} \cdot \frac{\partial v}{\partial x} d x \quad \text { for } u, v \in V,
$$

and

$$
B(t) v=(t+1)^{\theta} v .
$$

Then $A$ is the Fréchet derivative of a convex functional $F_{A}$ on $V$ defined by

$$
\begin{aligned}
F_{A}(u) & =\frac{1}{2} \int_{\Omega}\left|\frac{\partial^{2} u}{\partial x^{2}}\right|^{2} d x+\frac{a}{2} \int_{\Omega}\left|\frac{\partial u}{\partial x}\right|^{2} d x+\frac{1}{4}\left(\int_{\Omega}\left|\frac{\partial u}{\partial x}\right|^{2} d x\right)^{2} \\
( & \left.\equiv \frac{1}{2}\left|u_{x x}\right|_{H}^{2}+\frac{a}{2}\left|u_{x}\right|_{H}^{2}+\frac{1}{4}\left|u_{x}\right|_{H}^{4}\right) .
\end{aligned}
$$

When $\theta=0$ and $f \equiv 0$, Ball [1], [2] treated the initial value problem for (4.10) and showed the existence and uniqueness of solutions of (4.10). His technics can also apply to the case when $\theta \neq 0$ and $f \neq 0$; so that, for each $f \in L_{100}^{2}(0, \infty ; H), u_{0} \in V$ and $u_{1} \in H$, there exists a unique solution $u$ of (4.10) satisfying

$$
u(0)=u_{0} \text { and } u^{\prime}(0)=u_{1} .
$$

Moreover, such $u$ always satisfies the energy equation

$$
E(t)+\int_{0}^{t}(s+1)^{\theta}\left|u^{\prime}(s)\right|_{H}^{2} d s=E(0)+\int_{0}^{t}\left(f(s), u^{\prime}(s)\right)_{W} d s, \quad t \geqq 0
$$

where $E(t)=\frac{1}{2}\left|u^{\prime}(t)\right|_{H}^{2}+F_{A}(u(t))$.

Now we note

$$
\left|u_{x x}\right|_{H} \geqq \pi\left|u_{x}\right|_{H} \quad \text { for } u \in V \text {. }
$$

Thus

$$
F_{A}(u) \geqq \frac{1}{2}\left(a+\pi^{2}\right)\left|u_{x}\right|_{H}^{2}+\frac{1}{4}\left|u_{x}\right|_{H}^{4} \quad \text { for } u \in V .
$$

Therefore, for $a \geqq-\pi^{2}, A$ satisfies (A.1)' (see Remark 2.2) with $V_{1}=H_{0}^{1}(\Omega)$ and 


$$
\begin{cases}p=2 & \text { if } a>-\pi^{2} \\ p=4 & \text { if } a=-\pi^{2}\end{cases}
$$

Then, recalling Remark 3.1 we obtain the following results (cf. Ball and Carr [3]).

THEOREM 4.3. Let $a \geqq-\pi^{2},-1 \leqq \theta \leqq 1$ and let $u$ be a solution of (4.10)

(i) The case $a>-\pi^{2}$. If $|f(t)|_{H}=O\left(t^{-r}\right)$ with $\gamma>(1-\theta) / 2$ as $t \rightarrow \infty$, then

$$
E(t)=O\left(t^{-\alpha}\right) \quad \text { as } t \rightarrow \infty,
$$

where $\alpha$ is a positive number such that

$$
\begin{cases}\alpha \leqq 2 \gamma \text { and } \alpha<\frac{a+\pi^{2}}{2 \pi^{2}} & \text { if } \theta=1 \\ \alpha=2 \gamma-1+\theta & \text { if }-1<\theta<1 \\ \alpha \leqq 2 \gamma-2 \text { and } \alpha<\frac{2}{3} & \text { if } \theta=-1 .\end{cases}
$$

In particular, if $-1<\theta<1$ and $|f(t)|_{H}=O\left(\exp \left(-\gamma t^{1-|\theta|}\right)\right)$ with $\gamma>0$ as $t \rightarrow \infty$, then for some sufficiently small $\alpha>0$

$$
E(t)=O\left(\exp \left(-\alpha t^{1-|\theta|}\right)\right) \quad \text { as } t \rightarrow \infty .
$$

(ii) The case $a=-\pi^{2}$. If $|f(t)|_{H}=O\left(t^{-r}\right)$ with $\gamma>(1-\theta) / 2$ as $t \rightarrow \infty$, then

$$
E(t)= \begin{cases}O\left((\log t)^{-2}\right) & \text { as } t \rightarrow \infty \quad \text { if } \theta=1 \\ O\left(t^{-\alpha}\right) & \text { as } t \rightarrow \infty \quad \text { if }-1 \leqq \theta<1,\end{cases}
$$

where $\alpha$ is a positive number such that

$$
\begin{cases}\alpha=\min \{2(1-\theta), 2 \gamma-1+\theta\} & \text { if }-1<\theta<1 \\ \alpha \leqq \min \{4,2 \gamma-2\} \text { and } \alpha<2 / 3 & \text { if } \theta=-1 .\end{cases}
$$

Remark 4.3. Even if we replace the boundary conditions in (4.10) with the conditions corresponding to the case of clamped ends

$$
u=\frac{\partial u}{\partial x}=0 \quad \text { on } \Gamma \times[0, \infty)
$$


we can also investigate the decay property of solutions for such problems (which we denote by $\left.(4.10)^{\prime}\right)$.

Take $H=W=L^{2}(\Omega)$ and $V=H_{0}^{2}(\Omega)$. As for the existence of solutions of (4.10)' satisfying the energy equation (4.11), the same results as in the case of hinged ends hold true. (See [1] and [2].) However, we note that, instead of (4.12),

$$
\left|u_{x x}\right|_{H} \geqq 2 \pi\left|u_{x}\right|_{H}
$$

holds for every $u \in V$. Hence, for $a \geqq-4 \pi^{2}$, we can prove the similar results to those of Theorem 4.3 .

\section{REFERENCES}

[1] Ball, J. M., Initial-boundary value problems for an extensible beam, J. Math. Anal. Appl. 42 (1973), 61-90.

[2] - Stability theory for an extensible beam, J. Diff. Eqns. 14 (1973), 399-418.

[ 3 ] Ball, J. M. and J. Carr, Decay to zero in critical cases of second order ordinary differential equations of Duffing type, Arch. Rat. Mech. Anal. 63 (1976), 47-57.

[4] Barbu, V., Nonlinear semigroups and differential equations in Banach spaces, Noordhoff (1976).

[ 5 ] Brezis, H., Problèmes unilatéraux, J. Math. pures et appl. 51 (1972), 1-168.

[ 6 ] Caughey, T. K. and J. Ellison, Existence, uniqueness and stability of solutions of a class of nonlinear partial differential equations, J. Math. Anal. Appl. 51 (1975), $1-32$.

[ 7 ] Greenberg, J. M., On the existence, uniqueness, and stability of solutions of the equation $\rho_{0} X_{t t}=E\left(X_{x}\right)+\lambda X_{x x t}$, J. Math. Anal. Appl. 25 (1969), 575-591.

[ 8 ] Greenberg, J. M., R. C. MacCamy and V. J. Mizel, On the existence, uniqueness, and stability of solutions of the equation $\sigma^{\prime}\left(u_{x}\right) u_{x x}+\lambda u_{x t x}=\rho_{0} u_{t t}$, J. Math. Mech. 17 (1968), 707-728.

[9] Levine, H. A. and A. C. Murray, Asymptotic behavior and lower bounds for semilinear wave equations in Hilbert space with applications, SIAM J. Math. Anal. 6 (1975), 846-859.

[10] Lions, J. L., Quelques méthodes de résolution des problèmes aux limites non linéaires, Dunod Gauthier-Villars (1969).

[11] Lions, J. L. and W. A. Strauss, Some non-linear evolution equations, Bull. Soc. math. France 93 (1965), 43-96.

[12] Nakao, M., Convergence of solutions of the wave equation with a nonlinear dissipative term to the steady state, Memoirs Fac. Sci. Kyushu Univ. 30 (1976), 257-265.

[13] - Asymptotic stability of the bounded or almost periodic solution of the wave equation with nonlinear dissipative term, J. Math. Anal. Appl. 58 (1977), 336-343.

[14] - Decay of solutions of some nonlinear evolution equations, J. Math. Anal. Appl. 60 (1977), 542-549.

[15] - A difference inequality and its application to nonlinear evolution equations, J. Math. Soc. Japan 30 (1978), 747-762.

[16] Sattinger, D. H., Stability of nonlinear hyperbolic equations, Arch. Rat. Mech. Anal. 28 (1968), 226-244. 
[17] Strauss, W. A., On continuity of functions with values in various Banach spaces, Pacific J. Math. 19 (1966), 543-551.

[18] Tsutsumi, M., Some nonlinear evolution equations of second order, Proc. Japan Acad. 47 (1971), 950-955.

[19] Yamada, Y., Note on certain nonlinear evolution equations of second order, to appear.

Department of Mathematics

Faculty of Science

Nagoya University 